\title{
Correlation between Thermoelectric Properties and Plastic Deformation of p-Type $\mathrm{Bi}_{0.5} \mathrm{Sb}_{1.5} \mathrm{Te}_{3}$ Thermoelectric Materials
}

\author{
Kazuhiro Hasezaki ${ }^{1}$, Yoshinori Morisaki ${ }^{1, *}$, Hiroshi Araki ${ }^{1, *}$, \\ Hiroyuki Kitagawa ${ }^{1}$ and Eishi Tanabe ${ }^{2}$ \\ ${ }^{1}$ Department of Materials Science, Shimane University, Matsue, Shimane 690-8504, Japan \\ ${ }^{2}$ Western Hiroshima Prefecture Industrial Research Institute Manufacturing Technology Academy, Hiroshima 739-0046, Japan
}

\begin{abstract}
A plastic deformation process and ratio study of p-type $\mathrm{Bi}_{0.5} \mathrm{Sb}_{1.5} \mathrm{Te}_{3}$ was performed. The ingots were grown by the Bridgman method. Disks were cut from the ingots and deformed by either cold-pressing or by hot-pressing under pulse current heating. The plastic deformation ratio was controlled from 55 to $90 \%$. The crystal structures of the deformed samples were identified by X-ray diffraction and pole figure analyses. The diffraction patterns indicate that the surfaces and bottoms of the samples were highly oriented in the hexagonal (00- $l$ ) plane. Thermoelectric properties change depending on not the plastic deformation ratio but the kind of plastic deformation process. The power factor for hot-press deformed samples exceeded those for the original ingots and cold-press deformed samples. The results suggest that the process of hot-press deformation enhances the thermoelectric properties of p-type $\mathrm{Bi}_{0.5} \mathrm{Sb}_{1.5} \mathrm{Te}_{3}$.
\end{abstract}

(Received October 12, 2005; Accepted December 5, 2005; Published February 15, 2006)

Keywords: bismuth telluride, thermoelectric effects, plastic deformation, hot pressing

\section{Introduction}

Solid solutions of $\mathrm{Bi}_{2} \mathrm{Te}_{3}$ and $\mathrm{Sb}_{2} \mathrm{Te}_{3}$ have excellent $\mathrm{p}$ type thermoelectric properties and are the best materials for use in thermoelectric cooling and thermoelectric power generation near room temperature. ${ }^{1)}$ The crystal structure of these solid solutions is hexagonal (or rhombohedral) with the space group being $R 3 m,{ }^{2}$ ) where the lattice consists of a sequence of atomic layer units: - Te(1)-Bi-Te(2)-Bi-Te(1)along with a hexagonal $c$-axis. The bonds between $\mathrm{Bi}$ and $\mathrm{Te}$ are both ionic and covalent, and are quite strong. In contrast, Te-Te bonds are considered to result from Van der Waals type interactions, ${ }^{3)}$ and these bonds are extremely weak. Due to the presence of Van der Waals bonds, most of the physical properties of $\mathrm{Bi}_{2} \mathrm{Te}_{3}$ are known to be anisotropic. The thermoelectric properties are excellent along the $a$-axis. ${ }^{1,4)}$ On the other hand, cleavage is easily caused in melt-grown crystals, thus it is difficult to perform fine processing. Therefore, a structural control study seems necessary in this system. In a previous paper, authors reported on a plastic deformation study of $\mathrm{p}$ - and n-type $\mathrm{Bi}_{2} \mathrm{Te}_{3}$-related thermoelectric materials with various carrier concentrations.5) However, the optimum conditions for plastic deformation have not been clarified.

The present study investigated the correlation between thermoelectric properties and the plastic deformation process used for the ratio $\mathrm{Bi}_{0.5} \mathrm{Sb}_{1.5} \mathrm{Te}_{3}$, which is a typical p-type thermoelectric material. The source ingots were grown by the Bridgman method. The plastic deformation processes selected were cold pressing (CP) and pulse current hot pressing (PCHP). PCHP was performed by using spark plasma sintering (SPS) or plasma activated sintering (PAS) apparatus, that is, mechanical pressure and DC pulse current were applied to the samples and they were deformed just below the melting point. Formation of a texture having a preferred orientation could be expected from this process. The crystal

*Graduate Student, Shimane University structure and orientation were identified by X-ray diffraction and $\mathrm{X}$-ray pole figure analysis, and thermoelectric properties were evaluated by measuring the Hall coefficient, electrical resistivity and Seebeck coefficient. The effects of plastic deformation at room temperature are discussed.

\section{Experimental}

\subsection{Sample preparation}

The samples in the present study were p-type $\mathrm{Bi}_{0.5} \mathrm{Sb}_{1.5} \mathrm{Te}_{3}$, where 0.07 mass $\%$ Te was added in order to control the hole concentration. The raw materials $\mathrm{Bi}(6 \mathrm{~N})$, $\mathrm{Sb}(6 \mathrm{~N})$ and $\mathrm{Te}(6 \mathrm{~N})$ were weighed to obtain the target composition, and were heated for $4 \mathrm{~h}$ at $650^{\circ} \mathrm{C}$ under vacuum in a carbon-coated quartz ampoule, facilitating direct melting of the constituent elements. Crystal growth was then performed by means of the vertical Bridgman method (VBM) in an electric furnace with a temperature gradient, where the temperature gradient at the solidification point and the growth rate were estimated to be $0.4 \mathrm{~mm} / \mathrm{K}$ and $9 \mathrm{~mm} / \mathrm{h}$, respectively. The as-grown ingots were coarse-grained polycrystals which showed cleavage along the growth direction. The ingots were cut and shaped into cylinders or rectangular parallelepipeds parallel to the crystal growth direction.

Cold pressing (CP) was performed by applying a mechanical pressure of $250 \mathrm{MPa}$ at room temperature for $5 \mathrm{~min}$, with the pressure direction being vertical to the growth direction. Pulse current hot pressing (PCHP) was performed by using pulse current sintering apparatus. The ingot was placed on a graphite die. Mechanical pressure of $50 \mathrm{MPa}$ was applied to the ingot in the plane vertical to the growth direction through graphite punches. A pulse current of $400 \mathrm{~A}$ was applied and the temperature was raised to $400^{\circ} \mathrm{C}$ and held for $5 \mathrm{~min}$. The deformed samples were $10 \mathrm{~mm} \phi \times 1.5 \mathrm{~mm}(\mathrm{CP})$ and $11 \times$ $11 \times 1.5 \mathrm{~mm}(\mathrm{PCHP})$.

The deformation ratio $D_{\mathrm{R}}$ is defined by the following equation: 


$$
D_{\mathrm{R}} \equiv \frac{t_{0}-t}{t_{0}} .
$$

Where $t_{0}$ and $t$ indicate the initial thickness and deformed thickness, respectively. The plastic deformation ratio was varied by changing the initial shape of as-grown ingots within the range from 55 to $90 \%$.

\subsection{Evaluation of the crystal orientation (X-Ray dif- fraction and pole figure analysis)}

Structural investigation of the as-grown ingot and deformed sample surfaces was performed by X-ray diffraction (XRD) using $\mathrm{CuK} \alpha$ radiation in the range of $2 \theta=20-80 \mathrm{deg}$. The X-ray beams were radiated along the plane parallel to the growth direction for the VBM samples and the plane vertical to the pressure direction for the CP and PCHP samples. In $\mathrm{Bi}_{2} \mathrm{Te}_{3}$-related materials, the orientation of higher performance is $(00 \cdot l)$. Therefore, the orientation factor $F$ is defined as follows: ${ }^{6}$

$$
\begin{aligned}
F & \equiv \frac{\left(D-D_{0}\right)}{1-D_{0}} . \\
D & =\frac{\sum I_{00 l}}{\sum I_{h k l}} .
\end{aligned}
$$

where $\Sigma I_{00 l}$ indicates the sum of intensities from the $00 \cdot l$ plane, and $\Sigma I_{h k l}$ represents the sum of intensities within the measurement angle. $D_{0}$ represents $\Sigma I_{00 l}$ and $\Sigma I_{h k l}$ for the powder pattern. In the present paper, scanning was performed in the range of $2 \theta=20-80 \mathrm{deg}$, where $D_{0}$ becomes 0.03274 . According to eq. (2), $F$ is equal to 1 for complete $00 \cdot l$ orientation and 0 for non-oriented powder patterns.

Figure 1 is a schematic diagram of X-ray pole figure analysis. Measurement of (006) and (110) pole figures was carried out by Schulz's reflection method with a diffractom-

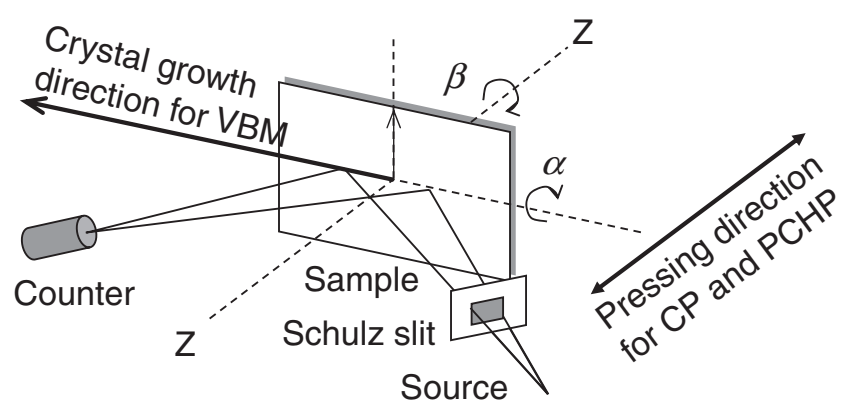

(a) X-ray pole figure analysis (XPMA)

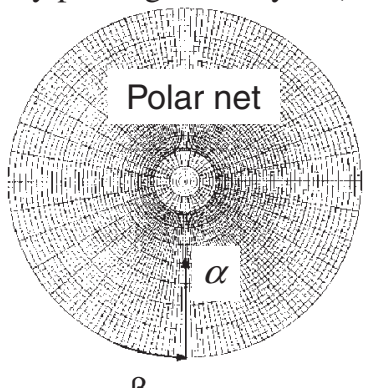

(b) Polar net

Fig. 1 Schematics of XPFA measurements (a) Sample setting (b) Polar net. eter. ${ }^{7)}$ The X-rays were radiated and rotated $\alpha$ and $\beta$ deg to the plane vertical to the pressure direction for the PCHP samples. The (006) and (110) pole figures are presented at $2 \theta=17.45$ and $45.15 \mathrm{deg}$, respectively. The step widths for step scanning of $\alpha(15<\alpha<90)$ and $\beta(0<\beta<360 \mathrm{deg})$ were $\Delta \alpha=1 \mathrm{deg}$ and $\Delta \beta=1 \mathrm{deg}$, respectively.

\subsection{Thermoelectric properties}

Thermoelectric properties were evaluated by measuring the Hall coefficient $R_{\mathrm{H}}$, electrical resistivity $\rho$ and the Seebeck coefficient $\alpha$ at room temperature. The Hall coefficient $R_{\mathrm{H}}$ and the electrical resistivity $\rho$ were measured by the van der Pauw method under a magnetic field of $0.5 \mathrm{~T}$ and an electric current of $50 \mathrm{~mA}$. The thermo-electromotive force $E$ was measured at a temperature difference $\Delta T$ of about $5 \mathrm{~K}$ and the Seebeck coefficient was estimated from $\alpha=E / \Delta T$.

\section{Results and Discussion}

\subsection{Crystal orientation}

Figure 2 shows the orientation factor on the surfaces of VBM, CP and PCHP samples estimated from eq. (2), plotted against the deformation ratio estimated from eq. (1). The plastic deformation using the $\mathrm{CP}$ and PCHP processes induced a preferred orientation in the crystals. The orientation factor does not show a clear dependence on the deformation ratio. However, the $a$-axis orientation factor increased for CP and PCHP.

Figure 3 shows pole figures of the highest orientations of PCHP samples in terms of the relative depth $(\mathrm{z})$ of the plane vertical to the pressing direction. Here, the relative depth is the position in the direction of thickness for samples when the surface and bottom are normalized as 0 and 1, respectively. (006) peaks at $\alpha=90 \mathrm{deg}$ were observed in all samples. The plastic deformation induced a preferred $(00-l)$ orientation of the crystals on the plane vertical to the pressure direction for the PCHP. Irregular (110) peaks were observed at all relative depths ( $\mathrm{z}$ ) of the plane vertical to the PCHP direction. This result shows that PCHP samples have coarse grains and an

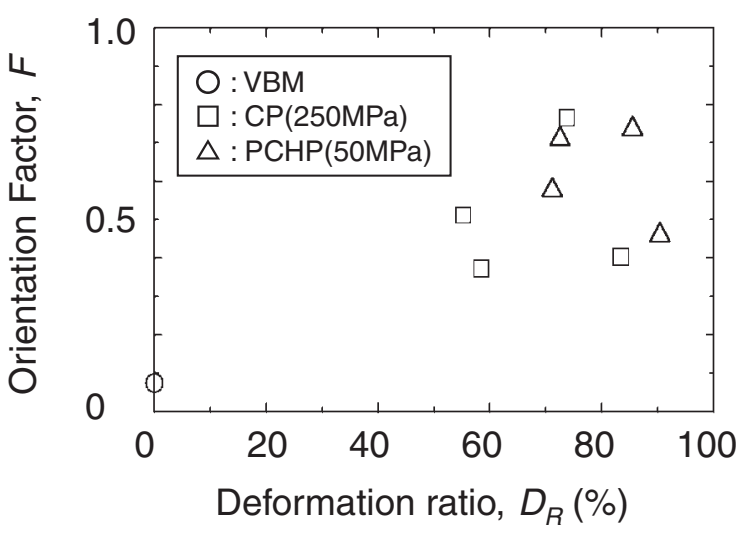

Fig. 2 Orientation factor on the surfaces of $\mathrm{p}$-type $\mathrm{Bi}_{0.5} \mathrm{Sb}_{1.5} \mathrm{Te}_{3}$ prepared by VBM, CP and PCHP methods plotted in terms of deformation ratio. (The direction of measurement for the VBM method was the plane parallel to the growth direction. The CP and PCHP methods were measured in the planes vertical to the $\mathrm{CP}$ and PCHP directions, respectively.) 

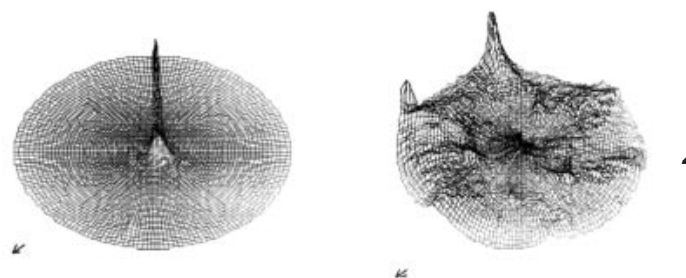

$z=0.23$
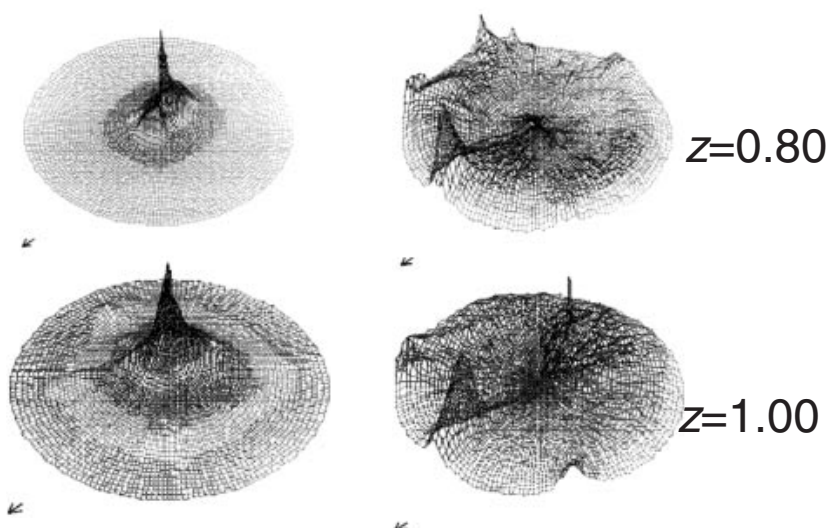

(b) $\left(\begin{array}{lll}1 & 1 & 0\end{array}\right)$ pole

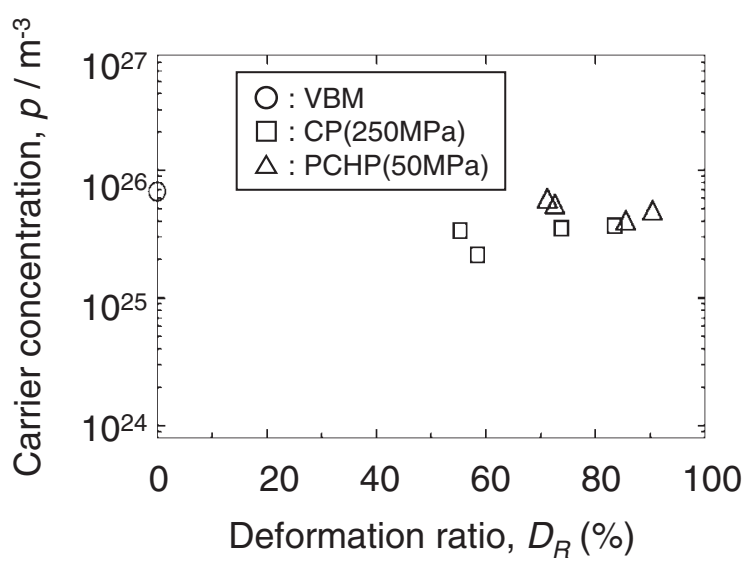

Fig. 5 Carrier concentration of p-type $\mathrm{Bi}_{0.5} \mathrm{Sb}_{1.5} \mathrm{Te}_{3}$ prepared by VBM, $\mathrm{CP}$ and PCHP methods plotted in terms of deformation ratio. (The direction of measurement for the VBM method was the plane parallel to the growth direction. The CP and PCHP methods were measured in the planes vertical to the $\mathrm{CP}$ and PCHP directions, respectively.)

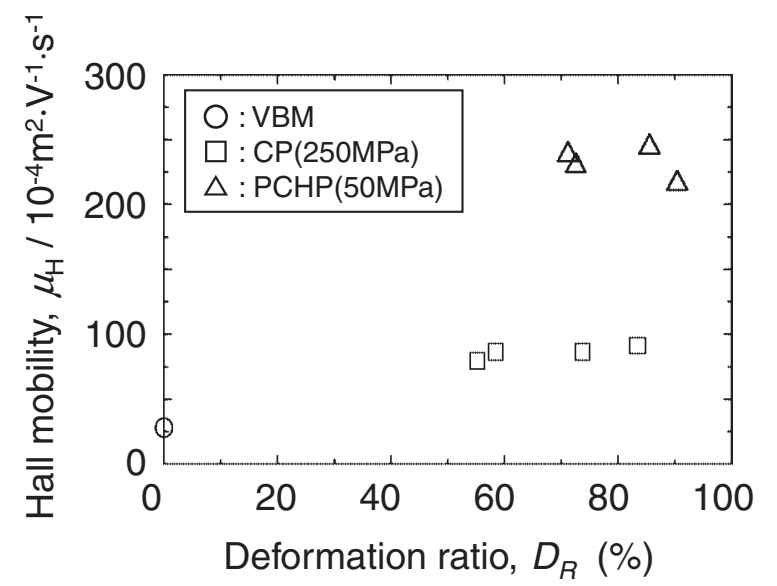

Fig. 6 Hall mobility of p-type $\mathrm{Bi}_{0.5} \mathrm{Sb}_{1.5} \mathrm{Te}_{3}$ prepared by VBM, CP and PCHP methods plotted in terms of deformation ratio. (The direction of measurement for the VBM method was the plane parallel to the growth direction. The CP and PCHP methods were measured in the planes vertical to the $\mathrm{CP}$ and $\mathrm{PCHP}$ directions, respectively.)

\subsection{Thermoelectric properties}

Figure 5 shows the relationship between carrier concentration at room temperature and the deformation ratio for $\mathrm{Bi}_{0.5} \mathrm{Sb}_{1.5} \mathrm{Te}_{3}$ prepared by means of VBM, CP and PCHP. The measurement direction was parallel to the plane of the growth direction for VBM samples and vertical to the plane of the pressure direction for CP and PCHP samples. The carrier (hole) concentration is almost constant, indicating that new carriers were not induced through the deformation processes. Furthermore, the carrier concentration is on the order of $10^{25} \mathrm{~m}^{-3}$ for all samples, which is suitable for thermoelectric conversion.

Figure 6 illustrates the relationship between Hall mobility at room temperature and the deformation ratio for $\mathrm{Bi}_{0.5^{-}}$ $\mathrm{Sb}_{1.5} \mathrm{Te}_{3}$ prepared by means of VBM, CP and PCHP. No clear dependence of the Hall mobility on the deformation ratio can be confirmed. On the other hand, the Hall mobility changes depending on the preparation process, that is, the affected by plastic deformation. 


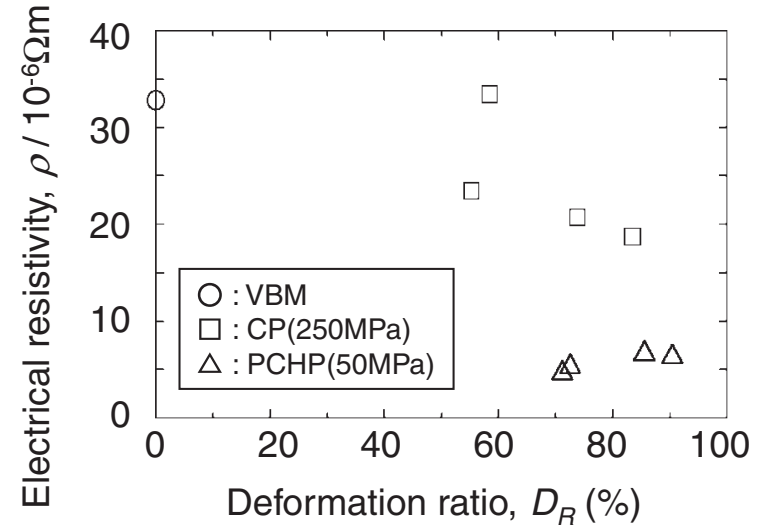

Fig. 7 Electrical resistivity of p-type $\mathrm{Bi}_{0.5} \mathrm{Sb}_{1.5} \mathrm{Te}_{3}$ prepared by $\mathrm{VBM}, \mathrm{CP}$ and PCHP methods plotted in terms of deformation ratio. (The direction of measurement for the VBM method was the plane parallel to the growth direction. The CP and PCHP methods were measured in the planes vertical to the $\mathrm{CP}$ and PCHP directions, respectively.)

Hall mobility of CP samples tends to increase more than that of VBM samples. This is considered to be related to the fact that the $a$-axis degree of orientation and Hall mobility both become large when plastic deformation takes place. ${ }^{5,8)}$ However, the level of the increase in the Hall mobility of the CP samples is insufficient. The Hall mobility of PCHP samples increases more than that of CP samples. This may be due to the removal of adverse defects that contribute to the Hall mobility by PCHP.

Figure 7 shows the relationship between the electrical resistivity $\rho$ of VBM, CP and PCHP samples and the deformation ratio at room temperature. The electrical resistivity $\rho$ is not substantially affected by the deformation ratio. However, $\rho$ changed with the preparation process. PCHP samples have the lowest electrical resistivity $\rho$, because PCHP samples have a large Hall mobility compared with VBM and CP samples. This is why the degree of orientation of the $a$-axis and the Hall mobility simultaneously become larger along with the removal of defects by PCHP.

Figure 8 shows the relationship between the Seebeck coefficient at room temperature and the deformation ratio for $\mathrm{Bi}_{0.5} \mathrm{Sb}_{1.5} \mathrm{Te}_{3}$ prepared by means of VBM, CP and PCHP. If the Seebeck coefficient increases with an increasing deformation ratio, it is attributed to anisotropy. It is well known that the anisotropy of the Seebeck coefficient is smaller than that of resistivity or thermal conductivity. ${ }^{9)}$ However, the Seebeck coefficient shows anisotropy due to the intrinsic conduction at room temperature. ${ }^{9)}$ No clear dependence of the Seebeck coefficient on the deformation ratio can be seen in Fig. 8.

Figure 9 illustrates the relationship between the power factor $\alpha^{2} \rho^{-1}$ at room temperature and the deformation ratio for $\mathrm{Bi}_{0.5} \mathrm{Sb}_{1.5} \mathrm{Te}_{3}$ prepared by means of VBM, CP and PCHP. The PCHP samples have a high power factor, because the Hall mobility improves along with the degree of orientation of the $a$-axis and the removal of defects by PCHP. The maximum power factor for VBM, CP and PCHP sample materials are estimated to be $0.55 \times 10^{-3} \mathrm{Wm}^{-1} \mathrm{~K}^{-2}$, $1.29 \times 10^{-3} \mathrm{Wm}^{-1} \mathrm{~K}^{-2}$ and $3.94 \times 10^{-3} \mathrm{Wm}^{-1} \mathrm{~K}^{-2}$, respectively.

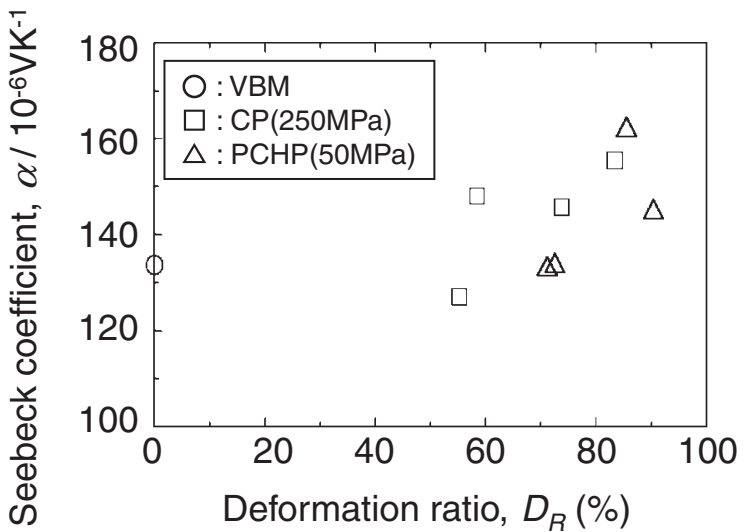

Fig. 8 Electrical resistivity of p-type $\mathrm{Bi}_{0.5} \mathrm{Sb}_{1.5} \mathrm{Te}_{3}$ prepared by VBM, CP and PCHP methods plotted in terms of deformation ratio. (The direction of measurement for the VBM method was the plane parallel to the growth direction. The $\mathrm{CP}$ and $\mathrm{PCHP}$ methods were measured in the planes vertical to the $\mathrm{CP}$ and $\mathrm{PCHP}$ directions, respectively.)

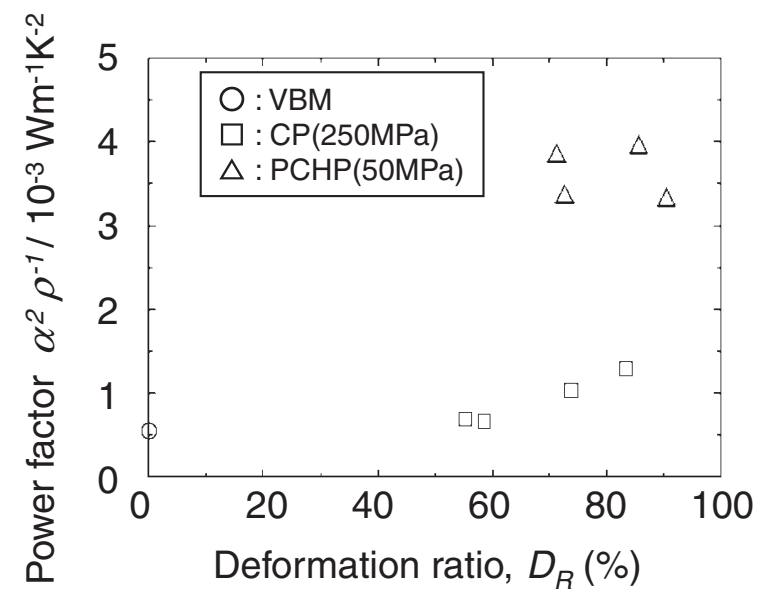

Fig. 9 Power factor of p-type $\mathrm{Bi}_{0.5} \mathrm{Sb}_{1.5} \mathrm{Te}_{3}$ prepared by VBM, CP and PCHP methods plotted in terms of deformation ratio. (The direction of measurement for the VBM method was the plane parallel to the growth direction. The $\mathrm{CP}$ and $\mathrm{PCHP}$ methods were measured in the planes vertical to the $\mathrm{CP}$ and PCHP directions, respectively.)

\section{Conclusion}

In the present study, source ingots were grown by the vertical Bridgman method (VBM). The ingots were deformed by cold pressing (CP) or pulse current hot pressing (PCHP) with various deformation ratios. The results are summarized as follows;

(1) The $a$-axis orientation was obtained in the plane vertical to the pressing direction for $\mathrm{CP}$ and PCHP samples. Pole figure measurements for PCHP samples show that the degree of $a$-axis orientation was imperfect.

(2) It is difficult to observe systematic differences in thermoelectric properties due to the deformation ratio. However, it is dependent on the preparation process.

(3) Strong anisotropy and removal of defects, i.e. useful thermoelectric properties, resulted from hot plastic deformation using the PCHP process. The power factor for PCHP samples was high $\left(3.94 \times 10^{-3} \mathrm{Wm}^{-1} \mathrm{~K}^{-2}\right)$ in the planes vertical to the pressing direction. 
It is concluded that PCHP is effective in obtaining a texture with $a$-axis orientation and removal of defects. As a result, thermoelectric properties are improved by the PCHP process.

\section{REFERENCES}

1) Ed. by D. Rowe: CRC Handbook of THERMOELECTRICS, (CRC Press, London, New York, Tokyo 1995) p. 239.

2) S. Nakajima: J. Phys. Chem. Solids. 24 (1963) 479.
3) J. Drabble and C. Goodman: J. Phys. Chem. Solids. 5 (1958) 142.

4) H. T. Kaibe, Y. Tanaka, M. Sakata and I. A. Nishida: J. Phys. Chem. Solids. 50 (1989) 945.

5) H. Kitagawa, Y. Morisaki, H. Ishibashi, M. Orihashi and Y. Noda: J. Japan Inst. Metal 69 (2005) 164-169.

6) F. K. Lotgering: J. Inorg. Nucl. Chem. 9 (1959) 113.

7) L. G. Schulz: J. Appl. Phys. 20 (1949) 1030.

8) H. T. Kaibe, Y. Tanaka, M. Sakata and I. A. Nishida: J. Phys. Chem. Solids. 50 (1989) 945-950.

9) H. Situmoranng and H. J. Goldsmid: Phys. Status Solidi B 134 (1986) K83-K88. 